\title{
Prevalência de talassemias e hemoglobinas variantes em pacientes portadores de lúpus eritematoso sistêmico \author{
erythematosus
} \\ Prevalence of thalassemias and variant hemoglobins in patients with systemic lupus
}

\author{
Frank S. Castro ${ }^{1}$ \\ Nilzio A. Silva ${ }^{2}$ \\ Paulo R. Melo-Reis ${ }^{3}$ \\ Karlla G. B. Dias-Penna ${ }^{3}$ \\ Cláudio B. Silva ${ }^{4}$ \\ Natália A. A. Brandão 5 \\ Rosane M. Cantero ${ }^{5}$ \\ Tatiana D. S. Ferreira ${ }^{5}$ \\ Lee C. Chen ${ }^{6}$
}

\begin{abstract}
O lúpus eritematoso sistêmico (LES) é uma doença tipicamente multigênica e multifatorial, com grande complexidade clínica e fisiopatológica. As causas do LES não são totalmente conhecidas, mas sabe-se que fatores ambientais e genéticos estão envolvidos. Dentre as várias manifestações clínicas observadas em pacientes com LES, as anemias chamam a atenção principalmente quando se observa nesse estudo uma prevalência de 52,5\% dos pacientes com índices hematimétricos sugestivos de anemias. Embora a anemia geralmente já seja observada em pacientes com LES, estudos sobre a prevalência de anemias hereditárias, especialmente as hemoglobinopatias na população com LES, não têm sido conduzidos. O objetivo desse trabalho foi o de avaliar a prevalência das hemoglobinopatias e talassemia em pacientes portadores de LES. Para isso, foram estudadas 80 amostras de sangue de pacientes portadores de lúpus atendidos no ambulatório do Hospital das Clínicas de Goiânia. Foram utilizados testes laboratoriais não moleculares para a detecção das hemoglobinopatias. A freqüência das alterações da hemoglobina foi de 10,0\%, encontradas em oito pacientes. Dessas alterações, a mais prevalente foi a talassemia alfa, encontrada em quatro pacientes, correspondendo a uma freqüência de 5,0\% da população estudada. Depois, foi o heterozigoto para a hemoglobina $S$, encontrada em dois pacientes, correspondendo a 2,5\% da população, e também outro heterozigoto para a hemoglobina $C$, encontrada em um paciente, correspondendo a 1,25\%, e um paciente com beta talassemia menor, correspondendo a 1,25\%. Nenhum caso de homozigose foi encontrado no presente estudo. Este trabalho demonstrou que não houve diferença na prevalência dos distúrbios da hemoglobina entre a população em geral e os portadores de LES. Rev. bras. hematol. hemoter. 2008;30(1):24-28.
\end{abstract}

Palavras-chave: Talassemia; hemoglobinas variantes; lúpus eritematoso sistêmico; diagnóstico laboratorial; HPLC.

${ }^{1}$ Professor e pesquisador associado ao Departamento de Biomedicina da Universidade Católica de Goiás.

${ }^{2}$ Professor do Depto. de Reumatologia, Universidade Federal de Goiás; Professor adjunto, Universidade Católica de Goiás, Depto. de Biomedicina. ${ }^{3}$ Professor assistente do Departamento de Biomedicina da Universidade Católica de Goiás.

${ }^{4}$ Laboratório de Análises Clínicas do Departamento de Biomedicina da Universidade Católica de Goiás.

${ }^{5}$ Aluna do Programa de Iniciação Científica - Pró-Reitoria de Pesquisa e Pós-Graduação da Universidade Católica de Goiás.

${ }^{6}$ Professora e pesquisadora do Laboratório de Radiobiologia e Mutagênese, Instituto de Ciências Biológicas, Departamento de Biologia Geral, Universidade Federal de Goiás.

Trabalho realizado no LEPAH - Laboratório de Estudo e Pesquisa de Anemias Hereditárias Prof. Dr. Paulo Cesar Naoum - Departamento de Biomedicina - Universidade Católica de Goiás.

Correspondência: Frank Sousa Castro

Universidade Católica de Goiás - Departamento de Biomedicina

Laboratório de Estudo e Pesquisa de Anemias Hereditárias Prof. Dr. Paulo Cesar Naoum (LEPAH) - Área IV - Bloco H - Sala 209

Av. Universitária 1069 - Setor Universitário

74605-010 - Goiânia-GO - Brasil

Tel.: (62) 3946-1195 - (62) 9968-6570

E-mail: knarfcastro@hotmail.com 


\section{Introdução}

O lúpus eritematoso sistêmico (LES) é uma doença autoimune, de etiologia desconhecida, multifatorial, que acomete o tecido conectivo de vários órgãos. ${ }^{1}$ Considerado como sendo uma das enfermidades do colágeno, ${ }^{2}$ o lúpus é uma doença auto-imune com diversidade de manifestações clínicas, que podem ser mucocutâneas e estar ou não acompanhadas de manifestações sistêmicas. ${ }^{3} \mathrm{O}$ aparecimento de auto-anticorpos está associado a uma falha na supressão de sua formação, que leva à produção de complexo antígenoanticorpo. Esse, por sua vez, deposita-se em diversos órgãos e responde pelo estado clínico do paciente. ${ }^{4}$

A doença lúpica é caracterizada por quatro ou mais dos critérios referendados pela então Associação Americana de Reumatismo. ${ }^{5}$ Esses critérios foram revistos por Hochberg, ${ }^{6}$ passando a utilizar os critérios de classificação propostos pelo American College of Rheumatology (ACR). Dentre os critérios referidos, as alterações hematológicas, principalmente as de série vermelha, que acometem a maioria dos pacientes com doença lúpica. Hochberg relata que $40 \%$ desses pacientes apresentam quadro de anemia. No eritrograma observa-se anemia leve ou moderada, valores do hematócrito até $30 \%$ abaixo do normal. ${ }^{6,7}$

As anemias hereditárias são as mais comuns e freqüentes doenças determinadas geneticamente. ${ }^{8} \mathrm{Na}$ população brasileira apresenta prevalências variáveis, pois depende da região do país onde é analisada e também do grupo étnico formador de cada uma. A miscigenação racial propiciou a dispersão dos genes anormais das talassemias e das hemoglobinas variantes. ${ }^{9,10}$ As hemoglobinas variantes $\mathrm{S}$ e C são as mais prevalentes na nossa população; quando em heterozigose, são assintomáticos, não desenvolvem doença e muitas vezes desconhecem sua condição., ${ }^{911,12,13}$ Já as talassemias constituem um grupo heterogêneo de doenças genéticas causadas pela redução da síntese de cadeias alfa e não alfa (beta, delta e gama) devido às deleções nos genes produtores de globina. ${ }^{15,16}$ A consequiência dessa falha de produção é a de provocar deficiência de hemoglobinização e os eritrócitos apresentam-se microcíticos e hipocrômicos. ${ }^{9,14,17}$

Dessa forma, a heterogeneidade genética e, conseqüentemente, a miscigenação progressiva da população brasileira facilitam a propagação e a interação entre as hemoglobinas variantes, talassemias, enzimopatias e esferocitoses. ${ }^{16,18}$ Entretanto, ainda não se conhece a prevalência de anemias hereditárias nesse grupo de portadores de doença lúpica. Portanto, o objetivo desse trabalho foi verificar a prevalência de hemoglobinas variantes e talassemias em pacientes portadores de LES em tratamento no Hospital das Clínicas de Goiânia.

\section{Casuística e Métodos}

O presente trabalho foi realizado após a aprovação do Comitê de Ética em Pesquisa da Universidade Católica de
Goiás, de acordo com normas da Resolução 196/98 do Conselho Nacional de Saúde e teve o consentimento livre e esclarecido de cada um dos participantes.

Foram coletadas oitenta amostras de sangue periférico, obtido por punção venosa, utilizando-se solução comercial de anticoagulante EDTA (10 g/dL), uma gota para cada $5 \mathrm{~mL}$ de sangue, provenientes de pacientes com diagnóstico prévio de LES e em tratamento no ambulatório do Departamento de Reumatologia do Hospital Universitário da Universidade Federal de Goiás, Goiânia-GO.

Os eritrogramas foram realizados no aparelho ABX Pentra ${ }^{\circledR} 60$, que quantificou o hematócrito (HCT), hemoglobina (HB), hemácias (RBC), os índices hematimétricos (VCM, HCM, CHCM) e de anisocitose (RDW).

A análise qualitativa das frações hemoglobínicas foi realizada por eletroforese em fita de acetato de celulose, $\mathrm{pH}$ alcalino, sendo que o hemolisado foi preparado com $50 \mu \mathrm{L}$ de sangue total e $100 \mu \mathrm{L}$ de solução de saponina a $1 \% \cdot{ }^{15} \mathrm{~A}$ confirmação e a quantificação das hemoglobinas normais e variantes foi realizada pelo sistema automatizado de cromatografia líquida de alta performance (HPLC), com equipamento Variant Bio-Rad e o programa ß-thalassemia short program. Outros testes auxiliares foram realizados: eletroforese em ágarfosfato $\mathrm{pH}$ ácido, dosagem de hemoglobina fetal, dosagem de hemoglobina $A_{2},{ }^{14,15}$ teste de falcização em solução de metabissulfito de sódio a $2 \%{ }^{19}$

A identificação da hemoglobina $\mathrm{H}$ foi realizada em eletroforese de hemoglobina em $\mathrm{pH}$ alcalino e pela pesquisa intra-eritrocitária ao microscópico óptico comum após incubação com solução de azul de cresil brilhante a $1 \%$ por sessenta minutos. ${ }^{20}$ Além desse, foram realizadas análise da morfologia dos eritrócitos, em esfregaço de sangue periférico, corado pelos corantes rápidos utilizados habitualmente em Hematologia Laboratorial. Observaram-se o tamanho e a cor dos eritrócitos, microcitose e hipocromia, respectivamente e também a forma. ${ }^{17}$ Todas as etapas do trabalho foram no período de maio a setembro de 2005.

\section{Resultados}

Dos oitenta pacientes portadores de LES atendidos no Hospital de Clínicas de Goiânia (HC), provenientes deste mesmo município e de outras regiões do estado de Goiás, 74 (92,5\%) eram do sexo feminino e seis (7,5\%), do masculino.

Constatou-se que 47,5\% (38 amostras) não apresentaram alterações no eritrograma, enquanto 52,5\% (42 amostras) dos pacientes analisados apresentavam alterações no eritrograma e índices hematimétricos compatíveis com anemia microcítica e hipocrômica. A Tabela 1 apresenta as médias e desvio padrão dos valores hematimétricos e concentrações de hemoglobinas para pacientes sem alterações no eritrograma, e a Tabela 2 apresenta os mesmos índices dos pacientes com alterações no eritrograma. 
Do total de oitenta amostras, oito (10\%) apresentaram hemoglobinas com alterações qualitativas ou quantitativas, sendo que sete (8,75\%) eram do sexo feminino e uma $(1,25 \%)$ do sexo masculino.

As metodologias empregadas nesta pesquisa permitiram a identificação de genótipos das talassemias e hemoglobinas variantes, sendo que todos foram de heterozigotos. A Tabela 3 ilustra os diferentes perfis hemoglobínicos identificados durante a realização desse trabalho.

Todas as amostras identificadas como portador de talassemia alfa pela presença de hemoglobina $\mathrm{H}$ em eletroforese de hemoglobina em acetato de celulose e em $\mathrm{pH}$ alcalino foram confirmadas pela pesquisa intra-eritrocitária de hemoglobina $\mathrm{H}$ e também pela quantificação dessa fração hemoglobínica.

A Tabela 4 mostra as distribuições dos valores do hematócrito e de hemoglobina dos pacientes que apresentaram valores abaixo dos valores referenciais para o sexo e idade.

Os heterozigotos tanto para hemoglobina $S$ (Hb AS) quanto para hemoglobina C (Hb AC) não apresentaram doença e nem alterações morfológicas no eritrograma. Entretanto, na análise morfológica do esfregaço sangüíneo corado desses pacientes constataram-se microcitose e hipocromia. ${ }^{17}$ Essas alterações não são esperadas para esses heterozigotos, por isso investigou-se a associação com alfa talassemia, a qual não foi detectada. ${ }^{29}$ Entretanto, não foi investigada a presença de ferropenia, o que poderia justificar a presença de microcitose e hipocromia.

\section{Discussão}

A população brasileira apresenta grande heterogeneidade genética caracterizada por significativa miscigenação racial. ${ }^{14,16}$ Dessa forma, a prevalência das hemoglobinopatias, provenientes de formas de hemoglobinas variantes e talassemias, variam de região para região e relacionam-se com os diversos grupos étnicos formadores de cada uma delas, fazendo da população brasileira única sob o ponto de vista antropológico. ${ }^{9}$ Por isso, é de fundamental importância ter essa consciência quando da abordagem ambulatorial de qualquer paciente, com ou sem anemia. ${ }^{9,23,24}$

O presente trabalho se propôs a investigar a prevalência de talassemias e hemoglobinas variantes em portadores de lúpus eritematoso sistêmico, uma vez que a maioria desses pacientes apresenta quadros persistentes de anemia leve a moderada, e também conhecer a prevalência de anemias hereditárias nesse grupo.
Tabela 1. Médias e desvio padrão dos valores hematimétricos e concentrações de hemoglobinas encontrados em 38 pacientes com eritrograma dentro dos limites da normalidade.

\begin{tabular}{cccccccc}
\hline & VCM & HCM & CHCM & RDW & Hb A & Hb A2 & Hb F \\
\hline Média & 87,6 & 29,1 & 33,0 & 14,0 & 96,78 & 2,67 & 0,05 \\
$\begin{array}{l}\text { Desvio } \\
\text { padrão }\end{array}$ & 3,7 & 1,7 & 1,1 & 1,9 & 4,40 & 0,35 & 0,18 \\
Referência & $87 \pm 5^{*}$ & $30 \pm 2^{*}$ & $34 \pm 2^{*}$ & $11-14 * *$ & $95-98 * *$ & $2,0-4,0$ ** & $0-1^{* *}$ \\
\hline
\end{tabular}

*Lorenzi ${ }^{21} ;{ }^{* *}$ Naoum $^{24}$

Tabela 2 . Médias e desvio padrão dos valores hematimétricos e concentrações de hemoglobinas encontrados em 42 pacientes com eritrograma apresentando alterações hematimétricas

\begin{tabular}{ccccccccccc}
\hline & VCM & HCM & CHCM & RDW & Hb A & Hb A2 & Hb F & Hb H & Hb C & Hb S \\
\hline $\begin{array}{l}\text { Média } \\
\text { Desvio }\end{array}$ & 77,8 & 25,4 & 32,7 & 14,6 & 50,6 & 1,5 & 0,1 & 4,8 & 37,5 & 35,7 \\
$\begin{array}{l}\text { padrão } \\
\text { Referência }\end{array}$ & 2,0 & 1,4 & 1,4 & 1,6 & 46,2 & 1,2 & 0,1 & 1,7 & - & 1,8 \\
\hline
\end{tabular}

* Lorenzi $^{21} ;{ }^{* *}$ Naoum $^{24}$

Tabela 3 . Distribuição das hemoglobinas normais e anormais em 80 amostras

\begin{tabular}{ccccccc}
\hline $\begin{array}{c}\text { Perfil } \\
\text { hemoglobínico }\end{array}$ & Feminino & $\%$ & Masculino & $\%$ & Totais & $\%$ \\
\hline AA & 67 & 83,75 & 5 & 6,25 & 73 & 90,0 \\
AC & 1 & 1,25 & 0 & 0,0 & 1 & 1,25 \\
AH & 4 & 5,0 & 0 & 0,0 & 4 & 5,0 \\
AS & 1 & 1,25 & 1 & 1,25 & 3 & 2,5 \\
Beta Tal menor & 1 & 1,25 & 0 & 0,0 & 1 & 1,25 \\
\hline Totais & 74 & 92,5 & 6 & 7,5 & 80 & 100 \\
\hline
\end{tabular}

Tabela 4. Distribuição dos valores do hematócrito e de hemoglobina dos pacientes que apresentaram valores abaixo dos referenciais ${ }^{21}$

\begin{tabular}{ccccc}
\hline & $\begin{array}{c}\text { Hematócrito } \\
\text { abaixo } \\
\text { dos valores } \\
\text { de referência }\end{array}$ & $\%$ & $\begin{array}{c}\text { Hemoglobina } \\
\text { abaixo } \\
\text { dos valores } \\
\text { de referência }\end{array}$ & $\%$ \\
\hline Masculino & 3 & 3,8 & 3 & 3,8 \\
Feminino & 39 & 48,8 & 35 & 43,8 \\
Total & 42 & 52,5 & 38 & 47,5 \\
$\begin{array}{c}\text { Referência } \\
\text { sexo masculino }\end{array}$ & $41-51 \%$ & & $14-16$ & \\
$\begin{array}{c}\text { Referência } \\
\text { sexo feminino }\end{array}$ & $37-47 \%$ & & $12-14$ & \\
\hline
\end{tabular}

Nas oitenta amostras analisadas encontramos um percentual de 47,5\% (38) com dosagem de hemoglobina abaixo do recomendado. ${ }^{21}$ Já o valor do hematócrito observou-se que $52,5 \%$ (42 amostras) estavam abaixo dos valores de referências para o sexo e a idade.

Constatou-se a prevalência de $10 \%$ de hemoglobinas variantes e de talassemias encontradas em oitenta pacientes portadores de LES, a qual está próxima aos valores encontra- 
dos em outros estudos, em populações não lúpicas. Em trabalho realizado com gestantes do estado de São Paulo, encontrou-se uma prevalência de hemoglobinas variantes e talassemias de 10,7\% da população analisada. ${ }^{25}$ Esses dados assemelham-se aos de Naoum et al., ${ }^{26}$ que encontraram um valor de $10 \%$, analisando 61 mil casos em todas as regiões brasileiras. Já no estudo realizado em estudantes no estado de Goiás encontrou-se uma freqüência de $10,1 \%{ }^{27}$ Assim, em nosso trabalho, apesar de analisar uma população diferenciada, ou seja, portadora de LES, os resultados obtidos não diferiram significativamente $(\mathrm{p}>0,05)$ dos autores acima citados, demonstrando dessa maneira que as anemias hereditárias apresentam distribuição equivalente nos mais variados grupos estudados.

Outro dado também concordante com esses autores é que a maior prevalência encontrada foi também talassemia alfa. Em nosso trabalho encontramos quatro pacientes, correspondendo a 5\% das oitenta amostras analisadas. Em alguns relatos observamos que as talassemias alfa constituem as mais comuns e prevalentes alterações hereditárias do homem. . $7,28^{27}$

Rothfield ${ }^{30}$ explica que os pacientes portadores de LES apresentam no curso da doença uma ou mais alterações hematológicas. Este quadro hematológico preeminente característico de anemia hemolítica é provocado por autoanticorpos que dificultam a investigação mais detalhada de um possível distúrbio da hemoglobina (estrutural ou funcional). Contudo, as características hematológicas nesse grupo nosológico variam muito e não obedecem a um determinado padrão. Portanto, há necessidade de estudos complementares nos portadores de LES nas diversas regiões brasileiras.

O presente estudo demonstrou que não foi constatada diferença significativa $(\mathrm{p}>0,05)$ na prevalência de talassemias e hemoglobinas variantes na população em geral e os portadores de LES.

\footnotetext{
Abstract

Systemic lupus erythematosus (SLE) is a typically multigenic and multifatorial disease with remarkable clinical and pathogenic complexities. The causes of SLE are not totally known, but It is known that environmental and genetic factors are involved. Among various clinical manifestations observed in lupus patients, anemia calls the attention because of a prevalence of $52.5 \%$ of the patients with $R B C$ indices suggestive of anemia identified in this study. Although anemia is usually seen in patients with SLE, studies of the prevalence of hereditary anemias, particularly hemoglobinopathies, have not been carried out in populations. The objective of this work was to evaluate the prevalence of hemoglobinophaties in patients with SLE. We studied 80 blood samples of patients with SLE in Hospital das Clínicas in Goiania, Brazil. The frequency of alterations of the hemoglobin was $10.0 \%$ (8 patients). Among these alterations, the most prevalent was alpha thalassemia in 4 patients $(5.0 \%$ of the studied population). The heterozygosity for hemoglobin $S$ was seen in 2 patients (2.5\%), hemoglobin C in one patient (1.25\%) and one
}

patient was identified with beta thalassemia minor. No homozygous cases were found in the present study. According to this work no difference in the prevalence of hemoglobin disorders was observed between general population and patients with SLE. Rev. bras. hematol. hemoter. 2008;30(1):24-28.

Key words: Thalassemia; variant hemoglobins; systemic lupus erythematosus; laboratorial diagnoses; HPLC.

\section{Referências Bibliográficas}

1. Antolín J, Amérigo MJ. Antecedentes históricos y conceptos actuales. En Lúpus eritematoso sistêmico. J. Font, M. Khamashta \& M. Vilardele (Orgs.), Menarini. Barcelona: 1996; 1-8.

2. Gahan E. Lupus erythematosus. Clinical observations in $443 \mathrm{ca}-$ ses. Arch Dermatol Syph. 1992;45:685.

3. McMurray RW et al. Sex hormones and systemic lupus erythematosus: review and meta-analysis. Arthritis Rheum. 2003;48(8):2100-10.

4. Cossermelli W et al. Lúpus eritematoso. São Paulo: Moreira Jr, 1978; 181p.

5. Tan CA et al. The 1982 revised criteria for the classification of systemic lupus erythematosus. Arthritis Rheum. 1982;25:1271-7.

6. Hochberg MC. Updating the American College of Rheumatology revised criteria for the classification of systemic lupus erythematosus. Letter. Arthritis Rheum. 1997:40:1725.

7. Carbotte $\mathrm{R}$ et al. Prevalence of cognitive impairment in systemic lupus erythematosus. J Nerv Ment Dis. 1986;174:357-64.

8. Wagner SC et al. Prevalência de talassemias e hemoglobinas variantes em pacientes com anemia não ferropênica. Rev. Bras. Hematol. Hemoter. 2005;27(1):37-42.

9. Orlando GM et al. Diagnóstico laboratorial de hemoglobinopatias em populações diferenciadas. Rev. Bras. Hematol. Hemoter. 2000; 22(2):111-21.

10. Naoum PC. Anemias imigrantes: a origem das anemias hereditárias no Brasil Ciência Hoje. 1984;3(14):59-64.

11. Melo-Reis PR et al. A importância do diagnóstico precoce na prevenção das anemias hereditárias. Rev. Bras. Hematol. Hemoter. 2006;28(2):149-52.

12. Weatherall DJ, Clegg JB. Inherited haemoglobin disorders: an increasing global health problem. Bullettin of the World Health Organization. 2001;79(8):704-12.

13. Zamaro PJ et al. Análise quantitativa e molecular de hemoglobina fetal em doadores de sangue. Rev. Bras. Hematol. Hemoter. 2002; 24(4):312-13.

14. Naoum PC. Hemoglobinopatias e Talassemias. Sarvier Ed. Livros Médicos. São Paulo, SP. 1997.

15. Naoum PC. Eletroforese. Técnicas e Diagnósticos. $2^{a}$ Ed. Santos Editora. São Paulo, SP. 1999.

16. Oliveira GL et al. Avaliação do perfil hematológico de portadores de talassemia alfa provenientes das regiões Sudeste e Nordeste do Brasil. Rev. Bras. Hematol. Hemoter. 2006;28(2):105-9.

17. Bain BJ et al. Diagnosis from the blood smear. N Engl J Med 2005; 353-507.

18. Backes CE et al. Triagem neonatal como um problema de saúde pública. Rev. Bras. Hematol. Hemoter. 2005;27(1):43-7.

19. Daland GA, Castle WB. A simple and rapid method for demonstrating sickling of the red blood cells: the use of reducing agents. Journal of Laboratory and Clinical Medicine. 1948;33:1082-8.

20. Ribeiro VS, Araújo JT. Hemoglobina H: Identificação laboratorial. Rev. Hosp. Clin. Fac. Med. S.Paulo. 1992;47(4):176-9. 
21. Lorenzi TF. Manual de Hematologia. Propedêutica e Clínica. 2a Ed. Medsi. São Paulo, SP. 1999, 641 p.

22. Naoum PC, Naoum FA. Hematologia Laboratorial - Eritrócitos. Edição da Academia de Ciência e Tecnologia. São José do Rio Preto-SP. 2005

23. Leoneli GG. et al. Hemoglobinas anormais e dificuldade diagnóstica. Rev. Bras. Hematol. Hemoter. 2000;22(3):396-403.

24. Naoum PC, Naoum FA. Doenças das Células Falciformes. Sarvier, 2004, 224p.

25. Viana-Baracioli LMS et al. Prevenção de hemoglobinopatias a partir do estudo em gestantes. Rev. Bras. Hematol. Hemoter. 2002; 23(1):31-9.

26. Naoum PC et al. Hemoglobinas no Brasil. Boletim 1986;141:180-8.

27. Melo-Reis PR. Avaliação da prevalência de hemoglobinas variantes e talassemias em Goiás: Métodos de identificação laboratorial e distribuição geográfica. Tese de mestrado, Mestrado de Ciências Ambientais e Saúde, Universidade Católica de Goiás. Goiânia-Goiás, 2004, 50p.

28. Naoum PC et al. Hemoglobinas anormais no Brasil. Prevalência e distribuição geográfica. Rev. Bras. Patol. Clin. 1987;23:68-78.

29. Tome-Alves $\mathrm{R}$ et al. Hemoglobinas AS/alfa talassemia: importância diagnóstica. Rev. Bras. Hematol. Hemoter. 2000;22(3):388-94.

30. Rothfield NF. Systemic lupus erythematosus: clinical and laboratory aspects. In Mc Carty D (ed): Arthritis and Allied Conditions, 11 th ed Philadelphia, Lea \& Febiger. 1989,1022-1047.

Avaliação: Editor e dois revisores externos

Conflito de interesse: não declarado

Recebido: 28/09/2006

Aceito após modificações: 07/05/2007 